\title{
NUMERICAL ASPECTS OF SINGULAR PERTURBATION PROBLEMS
}

by

\author{
P.W. Hemker \\ Numerical Mathematics Dept. \\ Mathematical Centre \\ Kruislaan 413 \\ Amsterdam, The Netherlands
}

\section{ABSTRACT}

In this paper a brief survey is given of the main problems that are encountered when singular perturbation problems are solved by numerical means. Some areas of current research are indicated.

For a two-dimensional model problem an error estimate is given for the Hughes and Brooks Streamline-Upwind Petrov-Galerkin method.

\section{INTRODUCTION}

Five or six years ago the field of singular perturbation problems was an almost undeveloped area of numerical analysis. Now the interest in it has grown and the number of papers devoted to the subject increases with considerable pace. Was the pioneering work by A.M. Il'in [35], Pearson [45], Dorr [21] and Kreiss et a1. [1] all devoted to difference schemes for two-point boundary-value problems, now the interest is shifting to 2-dimensional problems that are discretized mostly by finite-element type methods. The academic efforts approach more and more the engineering interests of methods for solving real-life problems such as the Navier-Stokes equations with large Reynolds number.

The existence, for small values of a parameter $\varepsilon$, of asymptotic expansions of the solution may do away with the necessity of special attention by numerical analysis. Indeed, the solution of the reduced problem, obtained after putting $\varepsilon=0$, can often be considered as a standard problem, as can the numerical computation of the boundary layer(s) on stretched coordinates. As soon as an asymptotic analysis is valid and a few terms in the asymptotic expansion describe the solution sufficiently accurate, one usually can rely on standard techniques to obtain numerical approximations.

However, numerical analysis of singular perturbation problems mainly concentrates on the following question: how to find a numerical approximation to the solution for small as well as for intermediate values of $\varepsilon$, where no short asymptotic expansion is available. Or, more general, how to construct a single numerical method that can be applied both in the case of extremely small $\varepsilon$ and for larger values of $\varepsilon$, when one wouldn't consider the problem as singularly perturbed any longer.

Here two main difficulties arise from the numerical point of view. (1) In the solution of singular perturbation problems often boundary layers appear, which may be 
very thin. In order to represent the solution by numerical means, a discretization of the region of definition $\Omega$ is required. E.g. a (regularly or irregularly distributed) set of points is chosen in $\Omega$, on which the solution is approximated. To represent the solution in the boundary layer either enough points should be placed in this layer or an a priori assumption about the shape of the boundary-layer solution is necessary. If the shape of the layer - or even its location - is unknown, this can yield an awkward situation. (2) The second difficulty arises in the case of elliptic singular perturbation problems, where - for larger values of $\varepsilon$ - all commonly used numerical methods make essential use of the ellipticity of the equation. If the reduced equation is no longer elliptic, the numerical method fails for small $\varepsilon$. In particular Finite Element Methods for the discretization of elliptic PDEs are well analyzed in the case of symmetric differential operators and small perturbations thereof. Many singular perturbation problems of interest, however, are given by essentially non-symmetric operators.

Numerical means to handle the first difficulty (mesh construction) are "adaptive discretization methods". Here, the proper mesh on which the differential problem is discretized, is generated during the process that computes the approximate solution. Thus, the processes of discretization and solution of the discretized equation are closely interwoven. For two-point boundary-value problems significant progress has been made in this direction [2,3,46]. Two general purpose codes for these problems, that can also be used for singularly perturbed problems, have been constructed by LentiniPereyra and by Ascher-Christiansen-Russel1 [17]. The development of numerical methods for adaptive discretization in two dimensions is only beginning. Thus far, a few attempts have been made for non-singular perturbation problems by Babuska and Rheinbold $[6,7]$. A program that implements an adaptive method is constructed by Bank et al. [10]. Some applications of the theory of Babuska to singular perturbation problems have been published by Reinhardt $[48,49]$. Apart from these results the subject seems to be completely open to research.

The emphasis in the numerical analysis of singular perturbation problems thus far lies with the 2nd difficulty: the construction and the analysis of methods for strongly non-symmetric operator equations. Here almost all research is devoted to 2 nd order elliptic equations with a significant first derivative. The model problems most1y studied are: in one dimension

$$
\begin{aligned}
& -\varepsilon y^{\prime \prime}+f y^{\prime}+g y=s, \quad \text { on } \Omega=(0,1), \\
& (1.1) \quad y(0)=\alpha, \quad y(1)=\beta, \\
& \varepsilon>0, \quad f, g, s \in C^{\circ}(\Omega) ;
\end{aligned}
$$

and in two dimensions 


$$
\begin{array}{ll}
-\nabla(\bar{\varepsilon} \nabla u)+\bar{b} \nabla u+c u=f & \text { on } \Omega \subset \mathbb{R}^{2} \\
u=g & \text { on } \Gamma_{D} \\
\bar{n} \bar{\varepsilon} \nabla u=h & \text { on } \Gamma_{N}
\end{array}
$$

$\Gamma_{\mathrm{D}} \cup \Gamma_{\mathrm{N}}=\partial \Omega, \bar{\varepsilon} \in \mathbb{R}^{2 \times 2}$ a positive definite matrix, $\overline{\mathrm{b}} \in\left[\mathrm{C}^{1}(\Omega)\right]^{2}$, with $\nabla \overline{\mathrm{b}}=0$ and $c, f \in \mathrm{C}^{\circ}(\Omega)$.

As was pointed out by Brandt [15], if the more-dimensional problem is the final aim, the one-dimensional problem is often a real over-simplification. In the first place this is by the dimensionality of the problem itself. In two dimensions the shape of the boundary-layers may be much more complex than in the one-dimensional case. The second reason is directly related to the numerical approximation of the equation. If the 1-D differential equation is approximated by a difference equation

$$
a_{i} y_{i-1}+b_{i} y_{i}, \quad c_{i} y_{i+1}=d_{i},
$$

the value $y_{i}$ is determined by the values $y_{i-1}$ and $y_{i+1}$, as is the case when the differential problem is restricted to the interval $\left[x_{i-1}, x_{i+1}\right]$. Hence, the coefficients in (1.3) can be determined such that $y_{i}$ is adproximated with an arbitrary high accuracy. This situation never occurs in the case of more dimensions, where the differential solution at a gridpoint is not determined by its value at any finite number of neighbouring gridpoints.

In this paper we first consider, in section 2, simple difference schemes for a 1-dimensional problem and we show what numerical difficulties arise when the differential problem becomes singularly perturbed. Here we indicate one line of research: the search for $\varepsilon$-uniform methods. In section 3 we introduce global methods: collocation, Galerkin and finite elements, and in the next section we show how error estimates for the usual methods degenerate for small $\varepsilon$. Here we mention the line of research which, in 2-dimensional problems, applies Petrov-Galerkin methods for adapting the schemes to singular perturbations. In section 5 we describe the Hughes and Brooks Petrov-Galerkin method and in section 6 we derive an error estimate for it. Finally we give some bibliographical notes and indicate some other lines of current research.

\section{FINITE DIFFERENCE METHODS}

The simplest example to show what happens with the discretization of strongly non-symmetric problems by classical means is given by the equation

$$
\left\{\begin{array}{l}
\varepsilon y^{\prime \prime}+y^{\prime}=0, \quad \text { on }(0,1) \\
y(0)=0, \quad y(1)=1 .
\end{array}\right.
$$

Central discretization on a regular mesh $\left\{0=x_{0}<x_{1}<\ldots<x_{N}=1 \mid x_{i}-x_{i-1}=h\right\}$ yields 


$$
\begin{aligned}
& L_{h, \varepsilon^{c}}^{c} y_{i} \equiv \varepsilon\left(y_{i+1}-2 y_{i}+y_{i-1}\right) / h^{2}+\left(y_{i+1}-y_{i-1}\right) / 2 h=0 \\
& y_{0}=0, \quad y_{N}=1 .
\end{aligned}
$$

Its solution reads

$$
y_{i}=\left(1-r^{i}\right) /\left(1-r^{N}\right)
$$

with $r:=(2 \varepsilon-h) /(2 \varepsilon+h)$. The exact solution of (2.1) at $x_{i}$ is given by (2.3) with $r:=\exp (-h / \varepsilon)$. We see that

$$
\left|\exp (-h / \varepsilon)-\frac{2 \varepsilon-h}{2 \varepsilon+h}\right|=O\left(\left(\frac{h}{\varepsilon}\right)^{3}\right) \text { for }\left(\frac{h}{\varepsilon}\right) \rightarrow 0
$$

and hence

$$
\left|y\left(x_{i}\right)-y_{i}\right|=O\left(\left(\frac{h}{\varepsilon}\right)^{2}\right) \quad \text { for }\left(\frac{h}{\varepsilon}\right) \rightarrow 0
$$

for a fixed point $x_{i}$ in $(0,1)$. The error is bounded by $c(\varepsilon) h^{2}$; i.e. the approximation is $2^{\text {nd }}$ order accurate, but the error constant $C(\varepsilon)$ may increase intolerably as $\varepsilon \rightarrow 0$. In fact we see that the discrete solution oscillates for $2 \varepsilon<h$, and (for even $N$ ) we find

$$
\begin{aligned}
\lim _{\varepsilon \rightarrow 0} y_{i} & =i / N \quad \text { (for even } i) \\
& \left.\approx \frac{h}{2 \varepsilon N} \quad \text { (for odd } i\right),
\end{aligned}
$$

whereas for the true solution $\lim _{\varepsilon \rightarrow 0} y\left(x_{i}\right)=0$. The large error for small $\varepsilon$ is clearly due to instability of the operator $I_{h, \varepsilon}^{c}$ since the eigenvalues of this discretized operator are

$$
\lambda_{i}=\frac{-2 \varepsilon}{h}+\frac{1}{h^{2}} \sqrt{4 \varepsilon^{2}-h^{2}} \cos \left(\frac{i \pi}{N+1}\right) .
$$

The simplest way to overcome this instability is to use "upstream" differences instead of central differences, i.e. to take

$$
\mathrm{I}_{\mathrm{h}, \varepsilon}^{\mathrm{u}} \mathrm{y}_{\mathrm{i}} \equiv \varepsilon\left(\mathrm{y}_{\mathrm{i}+1}-2 \mathrm{y}_{\mathrm{i}}+\mathrm{y}_{\mathrm{i}-1}\right) / \mathrm{h}^{2}+\left(\mathrm{y}_{\mathrm{i}+1}-\mathrm{y}_{\mathrm{i}}\right) / \mathrm{h}=0
$$

The solution of this difference equation is (2.3) with $r:=\varepsilon /(\varepsilon+h)$. Here we see

$$
\left|\exp (-\mathrm{h} / \varepsilon)-\frac{\varepsilon}{\varepsilon+h}\right|=O\left(\left(\frac{h}{\varepsilon}\right)^{2}\right)
$$

and hence, for a fixed $x_{i} \in(0,1)$, 


$$
\left|y\left(x_{i}\right)-y_{i}\right|=O\left(\frac{h}{\varepsilon}\right) \quad \text { for } \frac{h}{\varepsilon} \rightarrow 0
$$

This approximation is only $1^{\text {st }}$ order accurate for $h \rightarrow 0$, but here we see $\lim _{\varepsilon \rightarrow 0} y_{i}=0$ for $i>0$, i.e. the asymptotic behaviour of the true solution is reflected in its discrete approximation. This success of the "upstream" difference approximation depends crucially on the choice of the right one-sided difference, which uses the "upstream" value $y_{i+1}$ (i.e. away from the boundary layer). If we would have taken the difference approximation $\left(y_{i}-y_{i-1}\right) / h$ for $y^{\prime}(x)$, then we would not have found a good approximation to $y\left(x_{i}\right)$ at all.

We observe that

$$
\mathrm{L}_{\mathrm{h}, \varepsilon}^{\mathrm{u}}=\mathrm{L}_{\mathrm{h}, \varepsilon+\mathrm{h} / 2}^{\mathrm{c}}
$$

i.e. the "upstream" discretization is equivalent with the central discretization if we replace the parameter $\varepsilon$ by $\varepsilon+h / 2$. The addition of the extra term $+h / 2$ is called artificial diffusion or artificial viscosity.

The artificial viscosity formulation of the one-sided difference approximation can be used more generally for equation (1.1). Then the upstream discretization is equivalent with the central difference discretization, replacing $\varepsilon$ by

$$
\varepsilon+h 1 f\left(x_{i}\right) 1 / 2=\varepsilon+\operatorname{sign}\left(\frac{h f\left(x_{i}\right)}{2 \varepsilon}\right) \frac{f\left(x_{i}\right) h}{2} .
$$

\section{Uniform numerical methods for singular perturbation problems}

Both the error bounds (2.4) and (2.6) are of the form

$$
\left|y_{i}-y\left(x_{i}\right)\right| \leq c(\varepsilon) h^{p}
$$

the error is of order p, but the error constant depends on $\varepsilon$. In (2.4) the error really degenerates for $\varepsilon \rightarrow 0$, but also in (2.6) we cannot improve the bound so that $C(\varepsilon)$ is independent of $\varepsilon$. Indeed, if we set $i=1$ we find (II'in [35])

$$
\left|y_{1}-y\left(x_{1}\right)\right|=\left|\frac{1-\frac{\varepsilon}{\varepsilon+h}}{1-\left(\frac{\varepsilon}{\varepsilon+h}\right)^{N}}-\frac{1-\exp (-h / \varepsilon)}{1-\exp (-1 / \varepsilon)}\right|
$$

and

$$
\lim _{\substack{h \rightarrow 0 \\ \varepsilon=h}}\left|y_{1}-y\left(x_{1}\right)\right|=\frac{1}{2}-e^{-1} \not 0
$$

Thus, the error bound does not hold uniformly in $\varepsilon$. Il'in shows that an error bound 


$$
\left|y_{i}-y\left(x_{i}\right)\right| \leq C h \quad \text { for all } x_{i} \in \Omega_{h}
$$

with $C$ independent of $h$ or $\varepsilon$ is obtained for the discretization of (1.1) with $g(x)=0$, if central differences are used with an artificial diffusion:

$$
+\frac{f\left(x_{i}\right) h}{2}\left[\operatorname{coth}\left(\frac{f\left(x_{i}\right) h}{2 \varepsilon}\right)-\frac{2 \varepsilon}{f\left(x_{i}\right) h}\right] .
$$

This corresponds to the artificial diffusion (2.8) where sign( $z$ ) is replaced by $\operatorname{coth}(z)-1 / z$. The shape of this function is shown in figure 1 .

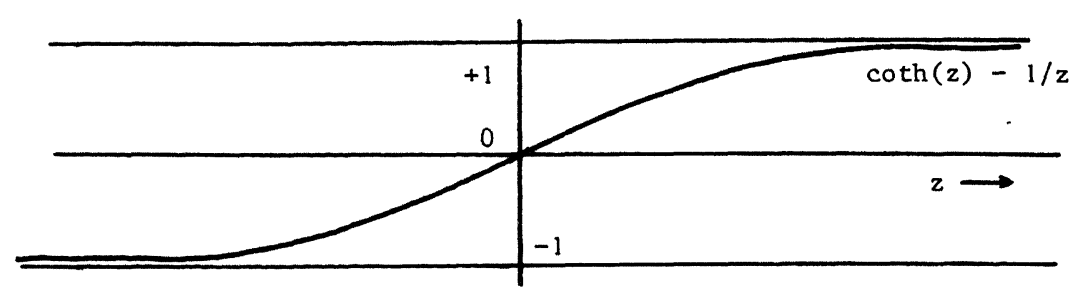

Figure 1. The exponential fitting function $\operatorname{coth}(z)-1 / z$.

Miller $[20,28,39]$ has proved that uniform bounds can be obtained only if the difference scheme is exponentially fitted, $i . e$. if the coefficients in the difference equations contain exponential functions.

Much effort has been spent on the construction and analysis of $\varepsilon$-uniform difference schemes and some results have been obtained. E.g. $2^{\text {nd }}$ order uniform methods have been constructed for the 1-dimensional convection diffusion equation (e.g. (1.1) with $b(x) \geq B>0, c(x) \leq 0)$, cf. $[14,20,23,28,37]$. However, fundamental difficulties arise when any of these methods are to be generalized to more dimensions and little progress has been reported so far.

3. GLOBAL METHODS

Systematic means for obtaining the discretization of a continuous equation

$$
\mathrm{Lu}=\mathrm{f},
$$

L a differential operator $L: S \rightarrow V, u \in S, f \in V, S$ and $V$ Banach spaces of functions defined over a region $\Omega$, are given by global or weighted residual methods. Here the solution $u$ of the continuous equation is approximated by an element $u_{h}$ in some finite $(\mathrm{N}-)$ dimensional function space $\mathrm{S}_{\mathrm{h}}$ the trial-space, $\mathrm{S}_{\mathrm{h}} \subset \mathrm{S}$. Since usually $\mathrm{u} \notin \mathrm{S}_{\mathrm{h}}$, $\mathrm{u}_{\mathrm{h}}$ is determined such that the residual, 


$$
f-\mathrm{Lu}_{\mathrm{h}} \text {, }
$$

is small is some sense. Examples of weighted residual methods are collocation methods where

$$
\left(f-L u_{h}\right)\left(x_{i}\right)=0
$$

is required for $\mathrm{N}$ points $\mathrm{x}_{i}$; or Galerkin methods where the requirement is

$$
\int\left(f-L u_{h}\right) \psi_{i} d \Omega=0
$$

for $\mathrm{N}$ functions $\psi_{i}$. The functions $\psi_{i}$ span the finite-dimensional function space $V_{h}$, the test-space. If $s_{h}=V_{h}$, the method is called a Bubnov-Galerkin method, if $s_{h} \neq V_{h}$ it is a Petrov-Galerkin method. Standard finite element methods (FEM) are BubnovGalerkin methods, where the functions $\psi_{i}$ have a small support in $\Omega$. The simplest FEM is constructed by taking a triangularization $T_{h}$ of the domain $\Omega$ with $N$ vertices $x_{i}$.

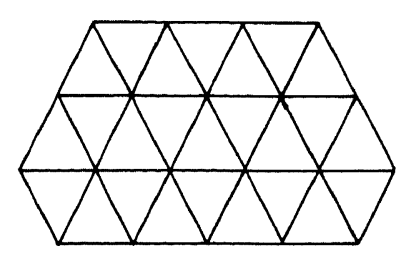

$T_{h}$ in $\Omega \subset \mathbb{R}^{2}$

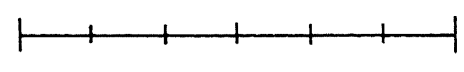

$T_{\mathrm{h}}$ in $\Omega \subset \mathbb{R}^{1}$.

To each $x_{i}$ a $\psi_{i}$ is chosen such that $\psi_{i}$ is linear over each triangle and $\psi_{i}\left(x_{j}\right)=\delta_{i j}$. For these $\psi_{i}$ we denote

$$
M^{0,1}\left(T_{h}\right)=\operatorname{Span}\left\{\psi_{i}\right\}
$$

Before the FEM discretization is applied to an elliptic differential equation $\mathrm{Lu}=\mathrm{f}$, where $L: c^{k+2 m}(\Omega) \rightarrow c^{k}(\Omega)$, this equation is reformulated to its weak form, where $L: H^{m}(\Omega) \rightarrow H^{-m}(\Omega)$, with $H^{m}(\Omega)$ and $H^{-m}(\Omega)$ the usual Sobolev spaces. Then the FEM uses $\mathrm{S}=\mathrm{V}=\mathrm{H}^{\mathrm{m}}(\Omega)$ and $\mathrm{S}_{\mathrm{h}}=\mathrm{V}_{\mathrm{h}} \subset \mathrm{H}^{\mathrm{m}}(\Omega)$.

\section{EXAMPLES}

The model equation (1.1) is reformulated as

$$
\begin{aligned}
& B(\psi, y)=\ell(\psi) \quad \text { for all } \psi \in \text { V with } \psi(0)=\psi(1)=0, \\
& y(0)=\alpha, y(1)=\beta, \\
& B(\psi, y)=\int_{0}^{1} \varepsilon \psi^{\prime} y^{\prime}+\psi f y^{\prime}+\psi g y d x, \\
& \ell(\psi)=\int_{0}^{1} \psi s d x .
\end{aligned}
$$


The 2-dimensional model equation (1.2) is reformulated as

(3.3)

$$
\begin{aligned}
& B(\psi, u)=\ell(\psi) \quad \text { for all } \psi \in V \text { with } \psi=0 \text { on } \Gamma_{D}, \\
& \mathbf{u}=g \quad \text { on } \Gamma_{D}, \\
& B(\psi, u)=\int \nabla \psi \bar{\varepsilon} \nabla u+\psi \bar{b} \nabla u+\psi c u d \Omega, \\
& \ell(\psi)=\int \psi f \mathrm{~d} \Omega+\int \psi h d \Gamma_{N} .
\end{aligned}
$$

For future reference we denote the latter problem also as: find $u \in S$ with $u=g$ on $\Gamma_{D}$ and

$$
\begin{aligned}
& B(\psi, u)=\ell(\psi) \quad \text { for all } \psi \in V_{B}, \\
& B(\psi, u)=(\nabla \psi, \bar{\varepsilon} \nabla u)+(\psi, \bar{b} \nabla u)+(\psi, c u), \\
& \ell(\psi)=(\psi, f)+\langle\psi, h\rangle_{N},
\end{aligned}
$$

$(\cdot, \cdot)$ and $<\cdot, \cdot\rangle_{N}$ denote the $L^{2}(\Omega)$ and the $L^{2}\left(\Gamma_{N}\right)$ innerproducts.

4. THE ONE-DIMENSIONAL PROBLEM

If we discretize equation (2.1), or rather its weak formulation

$$
\int_{0}^{1}-\varepsilon \psi^{\prime}(x) y^{\prime}(x)+\psi(x) y^{\prime}(x) d x=0,
$$

by the FEM with $S_{h}=v_{h}=M^{0,1}\left(T_{h}\right)$ on a regular partition $T_{h}=\left\{0=x_{0}<x_{1}<\ldots<x_{N}=\right.$ $1\}$ of $[0,1]$, then the discrete equation

$$
\begin{aligned}
& \sum_{j=0}^{N} \int_{0}^{1}-\varepsilon \psi_{i}^{\prime} \psi_{j}^{\prime}+\psi_{i} \psi_{j}^{\prime} d x u_{h}\left(x_{j}\right)=0, \\
& u_{h}(0)=0, \quad u_{h}(1)=1,
\end{aligned}
$$

is completely equivalent with eq. (2.2). Thus; we see that this FEM suffers from the same lack of stability as the central difference discretization.

In order to see this effect appear in a degenerating error estimate for a BubnovGalerkin method, when combined with the strongly asymetric bilinear form $B(v, u)$, we consider equation (1.1) with constant coefficients $\varepsilon, f, g \geq 0$ and homogeneous boundary conditions in its variational formulation (3.2). The solution satisfies

$$
B(v, u)=l(v) \quad \forall v \in v_{B} \text {; }
$$

the approximate solution $u_{h}$ satisfies 


$$
B\left(v_{h}, u_{h}\right)=\ell\left(v_{h}\right) \quad \forall v_{h} \in v_{h} \subset v_{B}
$$

Hence, the error $e=u_{h}-u$ satisfies

$$
B\left(v_{h}, e\right)=0 \quad \forall v_{h} \in v_{h}
$$

We easily derive

$$
\varepsilon l e^{\prime \|^{2}}+(e, g e)=B(e, e)=B\left(u-u_{h}, e\right)=B\left(u-v_{h}, e\right) \quad \forall v_{h} \in v_{h}=s_{h} \text {. }
$$

Further we find for $n=u-v_{h}^{\star}$, $v_{h}^{*}$ the best approximant in $s_{h}$ to $u$,

$$
\begin{aligned}
B(n, e)= & \left(n^{\prime}, \varepsilon e^{\prime}\right)+\left(\eta, f e^{\prime}\right)+(n, g e) \\
\leq \frac{\varepsilon}{2}\left\|\eta^{\prime}\right\|^{2} & +\frac{\varepsilon}{2}\left\|e^{\prime}\right\|^{2}+\frac{1}{2} g\|\|^{2}+\frac{1}{2} g\|\|^{2} \\
& +\frac{f}{2 p}\|\eta\|+\frac{p f}{2}\left\|e^{\prime}\right\| \quad \text { for all } p>0,
\end{aligned}
$$

whence

$$
(\varepsilon-p f)\left\|e^{\prime}\right\|^{2}+g\|\|^{2} \leq \varepsilon\|\eta\|^{2}+\left(g+\frac{f}{p}\right)\|\eta\|^{2} .
$$

To obtain a positive coefficient for $\mathrm{e}^{\prime l}$ we have to select $\mathrm{p}$ such that $\mathrm{pf} / \varepsilon=1 / \mathrm{c}<1$. We get

$$
\varepsilon\left(1-\frac{1}{c}\right)\left\|e^{\prime}\right\|^{2}+g\|\|^{2} \leq \varepsilon\left\|\eta^{\prime}\right\|^{2}+\left(g+f \frac{c f}{\varepsilon}\right)\|\eta\|,
$$

where $n$ is the error of the best approximant of $u$ in $S_{h}$. For large ratios $\frac{f}{\varepsilon}$ this cannot be a useful error estimate since the rhs is $O\left(\varepsilon^{-1}\right)$ for $\varepsilon \rightarrow 0$.

Several remedies have been proposed to obtain better global methods:

(i) to use artificial diffusion in the FEM discretization, i.e. to solve the problem for a value $\varepsilon$ which is increased up to $O(h)$;

(ii) to adapt the space $S_{h}$, such that the (rapidly changing) solution can be better approximated by elements from $s_{h}[41]$;

(iii) to adapt the space $v_{h}$ such that a stable discretization is found [30].

The first possibility is the simplest to apply, but it has the severe disadvantage that such a method can be only $1^{\text {st }}$ order accurate. The loss of accuracy is seen over the whole region, but becomes particularly apparent in the boundary layers which show up as overly diffusive. Hence, this approach was severely criticised for practical applications $[16,34]$. 
To obtain higher order methods that can be applied to problem (1.1), the adaptation of the trial- and test-space has been studied by several authors $[11,12,13,16$, $18,24,25,26,29,30,36,41,42,43]$. Almost all the analysis is made for the case where no turning points are present, i.e. $|f(x)| \geq c>0$. In this case a boundary layer appears only at the end of the interval and the boundary layer is of exponential type. Therefore, in order to fit the solution by an element of the trial space, the inclusion of exponential trial-functions in $S_{h}$ is a natural procedure $[13,41]$.

In order to obtain good pointwise approximations at the nodal points, it is advantageous to adapt the test-space. For the bounded bilinear form $B(v, u)$ and the Green's function $G(\cdot, \cdot)$ for problem $(1.1)$, we find

$$
\begin{aligned}
B\left(v_{h}, u-u_{h}\right) & =0 \quad \text { for all } v_{h} \in v_{h} ; \\
\left|\left(u-u_{h}\right)(x)\right| & =\left|B\left(G(x, \cdot), u-u_{h}\right)\right| \\
& =\left|B\left(G(x, \cdot)-v_{h}, u-u_{h}\right)\right| \leq c\left\|G\left(x_{i}\right)-v_{h}\right\| v\left\|u-u_{h}\right\| s ; \\
\left|\left(u-u_{h}\right)\left(x_{i}\right)\right| & \leq c\left\|u-u_{h}\right\| s \underset{v_{h} \in V_{h}}{\text { inf }}\left\|G\left(x_{i}, \cdot\right)-v_{h}\right\| \cdot .
\end{aligned}
$$

Hence, it is clear that pointwise errors are essentially smaller than the error in the $\|\cdot\|_{S}$-norm if the Green's function $G\left(x_{i}, \cdot\right)$ can be well approximated by elements in $\mathrm{V}_{\mathrm{h}}$.

The Green's functions $G\left(x_{i}, \cdot\right)$, being solutions of the adjoint of the original problem, show exponential boundary layers themselves (with the direction reversed). In practical methods, bases in $S_{h}$ and in $V_{h}$ are used of which all elements have a limited support. A typical basis of exponentially fitted spaces $S_{h}$ and $V_{h}$ is shown in figure 2. To obtain higher order approximations, these spaces can be supplemented with piecewise polynomials $[25,26,30]$.

Figure 2. A basis of exponentially fitted functions in $S_{h}$ and in $V_{h}$ for the equation $-\varepsilon y^{\prime \prime}+y^{\prime}=f$.
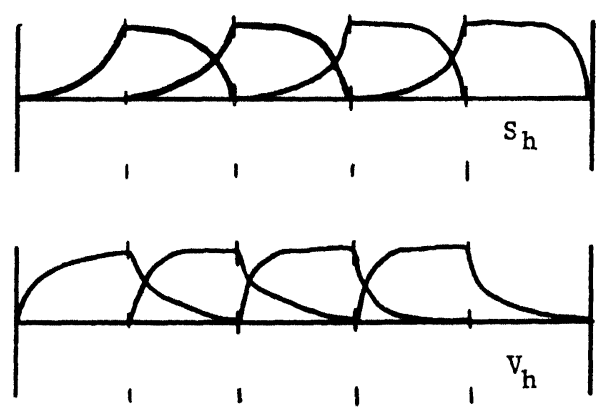

Both pointwise and global error-estimates, in the norm $\|$ el $\varepsilon=\sqrt{\varepsilon\left\|e^{\prime}\right\|^{2}+\| \text { e } \|^{2}}$, can be found in de Groen $[25,26]$. A further analysis based on the Generalized Lax-Milgram theorem 
is found in [8].

Other adaptations of the space of test-functions $\nabla_{h}$ are introduced by Christie et a1. [18], who use

$$
\begin{aligned}
& v_{h}=\left\{v \in H_{0}^{1}(a, b) \mid v=\sum_{j=1}^{N-1} v_{j}^{\phi}{ }_{j}^{\alpha}(x)\right\}, \\
& \phi_{j}^{\alpha}(x)=\left\{\begin{array}{l}
\left(x-x_{j-1}\right) / h_{j}+3 \alpha\left(x-x_{j-1}\right)\left(x_{j}-x\right) / h_{j}^{2}, \quad x \in\left(x_{j-1}, x_{j}\right), \\
\left(x_{j+1}-x\right) / h_{j+1}-3 \alpha\left(x-x_{j}\right)\left(x_{j+1}-x\right) / h_{j+1}^{2}, \quad x \in\left(x_{j}, x_{j+1}\right),
\end{array}\right.
\end{aligned}
$$

i.e. the piecewise linear functions are perturbed with piecewise quadratics. The parameter $\alpha$ is adjusted such that stable approximations are obtained. The selection of an optimal $\alpha$ is studied in [24].

Another Petrov-Galerkin method for the problem (1.1) is studied by Barrett and Morton $[11,12]$. They consider a test-space $v_{h}=N_{\varepsilon} S_{h}$, where $N_{\varepsilon}$ is an operator which approximately symmetrizes the bilinear form $B$, i.e. $N_{\varepsilon}$ is an approximation to the operator $\mathrm{N}: \mathrm{H}_{0}^{1} \rightarrow \mathrm{H}_{0}^{1}$ such that the operator $\mathrm{B}\left(\mathrm{Nw}_{1}, \mathrm{w}_{2}\right)$ is a symetric bilinear form.

\section{THE TWO-DIMENSIONAL PROBLEM}

We consider equation (1.2) and we study a Petrov-Galerkin method as was proposed by Hughes and Brooks [16]. For the trial-space we take $S_{h}=M^{0,1}\left(T_{h}\right)$ and for the testspace we use $\mathrm{V}_{h}=\tilde{M}_{h}$, where $\tilde{M}: H^{1}(\Omega) \rightarrow L_{2}(\Omega)$ is the operator defined by

$$
\tilde{M} u=u+k \bar{b} \nabla u, \quad k \in \mathbb{R}, \quad k \geq 0,
$$

with $\mathrm{k}=\mathrm{k}(\mathrm{h}, \varepsilon, \ldots)$. For the special case $\mathrm{k}=0$ the method is identical with the classical FEM and the method fails for singular perturbation problems. However, we search for a proper choice of $\mathrm{k}$ and derive error bounds that make sense also for sma11 $\varepsilon$. We see that for $S_{h}=M^{0,1}\left(T_{h}\right)$ the range $V_{h}=\tilde{M} S_{h}$ consists of functions that are the sum of a continuous, piecewise linear function $\psi$ and a discontinuous, piecewise constant function $\mathrm{k} \bar{b} \nabla \psi$.

Since $\nabla_{h} \notin H^{1}(\Omega)$ we have first to reformulate our original equation to another variational form than (3.3), before we can discretize it with this $V_{h}$. We construct an intermediate formulation between the classical form (1.2) and the weak form (3.3). By application of Green's formula over the domain $\Omega$, it is clear that any solution $u \in C^{2}(\Omega)$ of (1.2) is also a solution of (3.3); but, in general, if (3.3) admits a solution, (1.2) does not. Classical regularity theory, however, shows that for data smooth enough the solution of (3.3) is a solution of (1.2) indeed. In short, as soon as the existence of a solution of (1.2) and the uniqueness of the solution of (3.3) is established, the two are different formulations of one and the same problem.

For our formulation, let us consider an arbitrary partition of $\Omega$ in open 
subdomains $\left\{\Omega_{e}\right\}$ such that

$$
\bar{\Omega}=\bigcup_{e} \bar{\Omega}_{e} \quad \text { and } \quad \Omega_{e_{i}} \cap \Omega_{e}=\emptyset \quad \text { if } i \neq j,
$$

and the boundaries of $\Omega_{e}$ are piecewise smooth for each $\Omega_{e}$. We distinguish the following (disjunct) boundaries: the Dirichlet boundary $\Gamma_{D}$, the Neumann boundary $\Gamma_{N}$ and the internal boundary $\Gamma_{\text {int' }}$ such that

$$
\Gamma_{D} \cup \Gamma_{\mathrm{N}} \cup \Gamma_{\text {int }}=\underset{\mathrm{e}}{\cup} \partial \Omega_{\mathrm{e}}
$$

Let us now consider solutions $u \in H^{1}(\Omega) \cap \mathrm{C}^{\circ}(\bar{\Omega})$ of $(3.3)$ such that $\left.u\right|_{\Omega e} \in \mathrm{H}^{2}\left(\Omega_{\mathrm{e}}\right)$ for all $\Omega_{e}$. Possibly $u \notin c^{2}(\Omega)$ and hence equation $(1.2)$ has no classical meaning on $\Gamma_{\text {int }}{ }^{*}$ In order to interprete the weak problem in a classical sense, we formulate a condition over the inter-element boundaries that is satisfied by $u$. By definition, the solution $u$ of (3.2) satisfies

$$
(\nabla v, \bar{\varepsilon} \nabla u)+(v, \bar{b} \nabla u)+(v, c u)=(v, f)+\langle v, h\rangle
$$

$$
\text { for all } v \in H_{B}^{1}(\Omega)=\left\{u \in H^{1}(\Omega) \mid v=0 \text { on } \Gamma_{D}\right\}
$$

Since $\left.\mathrm{u}\right|_{\Omega e} \epsilon \mathrm{H}^{2}\left(\Omega_{\mathrm{e}}\right)$, we know for all $\mathrm{v} \in \mathrm{C}_{\mathrm{B}}^{1}(\Omega)=\left\{\mathrm{v} \in \mathrm{C}^{1}(\Omega) \mid \mathrm{v}=0\right.$ on $\left.\mathrm{I}_{\mathrm{D}}\right\}$,

$$
\sum_{e} \int_{\Omega e} v(-\nabla(\bar{\varepsilon} \nabla u)+\bar{b} \nabla u+c u) d \Omega_{e}=\sum_{e} \int_{\Omega_{e}}(\nabla v \bar{\varepsilon} \nabla u+v \bar{b} \nabla u+v c u) d \Omega_{e}
$$

$$
-\int_{\Gamma_{N}} \overline{v n \bar{\varepsilon}} \nabla u d \Gamma+\int_{\Gamma_{\text {int }}} j m p(\overline{v n \varepsilon} \nabla u) d \Gamma,
$$

where $\bar{n}$ is the outward normal on $\partial \Omega_{e}$, and on the boundary between $\Omega_{e}$ and $\Omega_{e}$ (i.e. for $x \in \bar{\Omega}_{e_{i}} \cap \bar{\Omega}_{e_{j}}$ ) we use the notation

$$
j m p(\overline{v n \varepsilon} \nabla u)(x)=-\sum_{k=i, j} \lim _{\substack{h \rightarrow 0 \\ x+h \in \Omega_{e}}} v_{e_{k}} \bar{n} e_{k} \bar{\varepsilon} \nabla u(x+h) .
$$

Equations (5.4) and (5.5) together with the boundary conditions on $\Gamma_{D}$ and $\Gamma_{N}$ yield

$$
\int_{\Gamma_{\text {int }}} j m p\left(v^{-} \bar{\varepsilon} \nabla u\right) d \Gamma=0 \text { for all } v \in C_{B}^{1}(\Omega),
$$

and hence $\bar{n} \bar{\varepsilon} \nabla u$ is continuous over inter-element boundaries.

Now we reformulate the problem (1.2) as:

find $u \in S=\left\{u\left|u \in H^{1}(\Omega) \cap C^{0}(\bar{\Omega}) ; u\right|_{\Omega_{e}} \in H^{2}\left(\Omega_{e}\right)\right\}$ such that 
$(5.7)$

(i) $\quad-\nabla(\varepsilon \nabla u)+\bar{b} \nabla u+c u=f$ on each $\Omega_{e}$;

(ii) $\overline{\mathrm{n}} \bar{\varepsilon} \nabla \mathrm{u}$ is continuous over inter-element boundaries

(iii) $u=g$ on $\Gamma_{D}$;

(iv) $\bar{n} \bar{\varepsilon} \nabla u=h$ on $\Gamma_{N}$.

It is immediately clear that a solution of (1.2) also satisfies (5.7) and that a solution of (5.7) is also a solution of (3.3).

The problem (5.7) can be written in a variational formulation as: find $u \in S$ such that $u=g$ on $\Gamma_{D}$ and

$$
\begin{aligned}
& \sum_{e}(w+v)(-\nabla(\bar{\varepsilon} \nabla u)+\bar{b} \nabla u+c u-f) d \Omega+\int_{\Gamma_{e}} \operatorname{vn} \bar{\varepsilon} \nabla u d \Gamma+ \\
& +\int_{\Gamma_{N}} v(\bar{n} \bar{\varepsilon} \nabla u-h) d \Gamma=0 \quad \text { for all } w \in L^{2}(\Omega) \text { and all } v \in H_{B}^{1}(\Omega) .
\end{aligned}
$$

Taking $w=M v, M: H_{B}^{1}(\Omega) \rightarrow L^{2}(\Omega)$ an injection, we can write this variational formulation as: find $u \in S$ such that for all $v \in H_{B}^{1}(\Omega)$

$$
\tilde{\mathrm{B}}(\mathrm{v}, \mathrm{u})=\tilde{\ell}(\mathrm{v}),
$$

where

$$
\begin{aligned}
& \tilde{B}(v, u)=(M v,-\nabla(\bar{\varepsilon} \nabla u))+(\nabla v \bar{\varepsilon} \nabla u)+(v+M v, \bar{b} \nabla u+c u), \\
& \tilde{\ell}(u)=(v+M v, f)+\langle v, h\rangle_{N} .
\end{aligned}
$$

This variational form is discretized to obtain the Hughes and Brooks Petrov-Galerkin method: the trial-space is $S_{h}=M^{0,1}\left(T_{h}\right) \subset S$ and the test space is $V_{h}=S_{h} \cap H_{B}^{1}(\Omega)$. Further $M: H_{B}^{1}(\Omega) \rightarrow L^{2}(\Omega)$ is chosen to be

$$
M v=k \bar{b} \nabla v \quad \text { for } a 11 \quad v \in H_{B}^{1}(\Omega)
$$

\section{AN ERROR ESTIMATE FOR THE PETROV-GAIERKIN METHOD}

We consider the problem (5.7) or (5.8) with the additional assumptions

(6.1)

$$
\begin{array}{ll}
\bar{\varepsilon} \in \mathbb{R}^{2 \times 2} & ; \bar{\varepsilon} \text { positive definite; } \\
\overline{\mathrm{b}} \in\left[\mathrm{H}^{1}(\Omega)\right]^{2} & ; \nabla \overline{\mathrm{b}}=0 ; \\
\mathrm{c} \in \mathrm{C}^{\mathrm{O}}(\Omega) & ; 0 \leq \mathrm{C}(\mathrm{x}) ; \\
\mathrm{f} \in \mathrm{L}^{2}(\Omega) & ; \\
\mathrm{g} \text { such that a } \tilde{\mathrm{g}} \in \mathrm{H}^{2}(\Omega) \text { exists for which }\left.\tilde{\mathrm{g}}\right|_{\Gamma_{\mathrm{D}}}=\mathrm{g} ; \\
\mathrm{h} \in \mathrm{L}^{2}\left(\mathrm{r}_{\mathrm{N}}\right) & ; \overline{\mathrm{n} b} \geq 0 \text { on } \mathrm{r}_{\mathrm{N}} .
\end{array}
$$


However, for simplicity in the formulations to follow, we restrict ourselves further to constant $c$ and $k$. By $C$ we denote a generic constant which is independent of $h$ or $\varepsilon$. To derive an error estimate for the Hughes- and Brooks Petrov-Galerkin method, we denote the error as $e=u_{h}-u$, where $u$ and $u_{h}$ satisfy

and

$$
\tilde{B}(v, u)=\tilde{l}(v) \quad \text { for all } v \in H_{B}^{1}(\Omega) \text {, }
$$

$$
\tilde{B}\left(v_{h}, u_{h}\right)=\tilde{l}\left(v_{h}\right) \quad \text { for a11 } v_{h} \in v_{h} \subset H_{B}^{1}(L) \text {. }
$$

Hence

$$
\tilde{B}\left(v_{h}, e\right)=0 \text { for all } v_{h} \in V_{h} \text {. }
$$

Analogous to $(4.3)-(4.4)$ we find

$$
\tilde{B}(e, e)=\tilde{B}\left(u_{h}-u+v_{h}, e\right)=\tilde{B}\left(v_{h}-u, e\right)=\tilde{B}(n, e) .
$$

With the norms $\|u\|=\sqrt{(u, u)}$ (the $L^{2}(\Omega)$ norm) and $\|\nabla u\|_{E}=\sqrt{(\nabla u, \bar{\varepsilon} \nabla u)}$, we get, for any $t, p>0$.

$$
\begin{aligned}
\tilde{B}(n, e)=-(k \bar{b} \nabla \eta, \nabla(\bar{\varepsilon} \nabla u))+(\nabla \eta \bar{\varepsilon} \nabla u)+(k \bar{b} \nabla \eta+\eta, \bar{b} \nabla e+c e) \\
=-(k \bar{b} \nabla \eta, \nabla(\bar{\varepsilon} \nabla u))+t\left[\|\nabla e\|_{E}^{2}+\frac{k+p}{2}\|\bar{b} \nabla e\|^{2}+c\|e\|^{2}\right] \\
+\frac{1}{2 t}\left[\frac{1}{2}\|\eta\|_{E}^{2}+k(1+k c)\|\bar{b} \nabla \eta\|^{2}+\left(c+\frac{1}{p}\right)\|\eta\|\right] .
\end{aligned}
$$

Similarly we derive

$$
\tilde{B}(e, e)=-(k \bar{b} \nabla e, \nabla(\bar{\varepsilon} \nabla e))+\|\nabla e\|_{E}^{2}+k\|\bar{b} \nabla e\|^{2}+c\|e\|^{2}+(1+k c)(e, \bar{b} \nabla e) .
$$

Using $e=0$ on $\Gamma_{D}$ and $\bar{n} \bar{b} \geq 0$ on $\Gamma_{N}$, we see

$$
(1+k c)(e, \bar{b} \nabla e)=\frac{1+k c}{2}<\bar{n} \bar{b} e^{2}>\geq 0
$$

Combination of $(6.2)-(6.5)$ with $t=\frac{1}{2}$ yields

$$
\begin{aligned}
& \frac{1}{2}\left(\|\nabla e\|_{E}^{2}+c\|e\|^{2}+(1+k c)<\bar{n} \bar{b} e^{2}>\right)+\frac{1}{4}(3 k-p)\|\bar{b} \nabla e\|^{2} \\
& =(k \bar{b} \nabla e, \nabla(\bar{\varepsilon} \nabla u))-(k \bar{b} \nabla \eta, \nabla(\bar{\varepsilon} \nabla u))+\frac{1}{2}\|\nabla \eta\|_{E}^{2}+k(1+k c)\|\bar{b} \nabla \eta\|^{2}+\left(c+\frac{1}{p}\right)\left\|_{\eta}\right\|^{2} .
\end{aligned}
$$

Now we use the well-known approximation result [19] 


$$
\inf _{u_{h} \in S_{h}}\left\|u-u_{h}\right\|+h\left\|\nabla u-\nabla u_{h}\right\| \leq C h^{2}\|u\|_{2},
$$

with $\|\cdot\|_{2}$, the norm in $H^{2}(\Omega)$, to obtain

$$
\|\mathrm{e}\|\left\|^{2}:=\right\| \nabla \mathrm{e}\left\|_{\mathrm{E}}^{2}+c\right\|\left\|^{2}+(1+\mathrm{kc})\left\langle\overline{\mathrm{nb}} \mathrm{e}^{2}\right\rangle+\frac{1}{2}(3 \mathrm{k}-\mathrm{p})\right\| \bar{b} \nabla \mathrm{v} \|
$$

$$
\leq 2 \mathrm{k} \varepsilon_{\mathrm{m}}\|\bar{b} \nabla e\|\|u\|_{2}+\mathrm{clu} \|_{2}^{2}\left\{\mathrm{~kb}{ }_{\mathrm{m}} \varepsilon_{\mathrm{m}} \mathrm{h}+\mathrm{h}^{2}\left(\varepsilon_{\mathrm{m}}+\mathrm{kb}_{\mathrm{m}}+\left(\mathrm{c}+\frac{1}{\mathrm{p}}\right) \mathrm{k}^{2}\right)\right\},
$$

where $\varepsilon_{\mathrm{m}}$ is the greater eigenvalue of $\bar{\varepsilon}$,

$$
b_{m}=\max \left(\left\|b_{1}\right\|_{L^{\infty}(\Omega)},\left\|b_{2}\right\|_{L^{\infty}(\Omega)}\right) .
$$

From (6.6), for $\bar{b}=0$, we derive the error estimate for the symmetric case:

$$
\|\nabla e\|_{E}^{2}+c\|e\|^{2} \leq \mathrm{Ch}^{2}\left(\varepsilon_{\mathrm{m}}+\mathrm{h}^{2}\right)\|u\|_{2}^{2} .
$$

If $\vec{b} \neq 0$ we find the estimate for the asymmetric case. With $k \neq 0$ we recover the estimate for the ordinary FEM:

$$
\|\nabla e\|_{E}^{2}+c\|e\|^{2}-\frac{p}{2}\|\bar{b} \nabla e\|^{2}+\left\langle\bar{n} \bar{b} e^{2}>\leq C h^{2}\left[\varepsilon_{m}+\left(c+p^{-1}\right) / h^{2}\right]\|u\|_{2}^{2} .\right.
$$

In order that $c\|\nabla e\|_{E}^{2}<\|\nabla e\|_{E}^{2}-\frac{p}{2}\|\bar{b} \nabla e\|^{2}$ we have to select $p$ such that

$$
\frac{\mathrm{p}}{2}<\frac{\varepsilon_{\min }}{\mathrm{b}_{\mathrm{m}}^{2}} ;
$$

$\varepsilon_{\min }$ the smaller eigenvalue of $\varepsilon$. Hence, the error bound is proportional to $\varepsilon_{\min }^{-1}$ for small values of $\varepsilon_{\min }$.

However, if $\mathrm{k} \neq 0$ we may set $\mathrm{p}=\mathrm{k}$ and obtain

$$
\begin{aligned}
& \|\mathrm{e}\|^{2}=\|\nabla e\|_{\mathrm{E}}^{2}+\mathrm{k}\|\bar{b} \nabla \mathrm{e}\|^{2}+\mathrm{c}\|\mathrm{e}\|^{2}+(1+\mathrm{kc})<\overline{\mathrm{n}} \overline{\mathrm{b}} \mathrm{e}^{2}> \\
& \leq 2 \sqrt{\mathrm{k}} \varepsilon_{\max }\|\mathrm{e}\|\|\mathrm{u}\|_{2}+\mathrm{C}\left\{\mathrm{kb} \mathrm{m}_{\mathrm{m}} \varepsilon_{\mathrm{m}} \mathrm{h} \mathrm{h}^{2}\left[\varepsilon_{\mathrm{m}}+\mathrm{kb} \mathrm{b}_{\mathrm{m}}+\left(\mathrm{c}+\frac{1}{\mathrm{k}}\right) \mathrm{h}^{2}\right]\right\}\left\|_{\mathrm{u}}\right\|_{2}^{2},
\end{aligned}
$$

from which we derive (assuming $b_{m}<C$ )

$$
\|\mathrm{e}\|=\sqrt{\mathrm{k}} \varepsilon\|\mathrm{u}\|_{2}+\|u\|_{2} \sqrt{\mathrm{k} \varepsilon_{\mathrm{m}}^{2}+C\left\{\varepsilon_{\mathrm{m}} \mathrm{kh}+\mathrm{h}^{2} \varepsilon_{\mathrm{m}}+\mathrm{h}^{2} \mathrm{k}+\mathrm{h}^{4} \mathrm{c}+\mathrm{h}^{4} / \mathrm{k}\right\}} .
$$

For $\varepsilon_{\mathrm{m}} \leq \mathrm{h}$ we find

$$
\| \text { ell } \leq \text { cll }{ }_{2} \sqrt{\mathrm{kh}^{2}+\mathrm{h}^{3}+\mathrm{h}^{4} c+\mathrm{h}^{4} / \mathrm{k}} \text {. }
$$

Hence, for small $\varepsilon_{\mathrm{m}} \leq O(h)$, an optimal bound is found for $k=O(h)$; then we find 


$$
\|\mathrm{e}\| \mathrm{Ch}^{3 / 2} \|_{2} \text {. }
$$

For $\varepsilon_{\mathrm{m}} \sim 1$ we find

$$
\| \text { ell } \leq \mathrm{Clul} 2 \sqrt{\mathrm{k}+\mathrm{h}^{2}+\mathrm{h}^{4} / \mathrm{k}} \leq \mathrm{Ch}\|\|_{2},
$$

for the optimal $k=O\left(h^{2}\right)$. For this $\varepsilon_{m}$ and $k$, the errorbound is as sharp as in the classical case with $b=0$ (cf. eqn. (6.7)). For $\varepsilon_{m} \approx 1$, if we assume $c_{1} h^{2} \leq k \leq c_{2} h$, we find

$$
\left\|\mathrm{el} \leq \mathrm{Clull} 2 \sqrt{\mathrm{ke} \varepsilon_{\mathrm{m}}^{2}+\mathrm{h}^{2} \varepsilon+\mathrm{h}^{4} / \mathrm{k}} \leq \mathrm{Ch}\right\| \mathrm{ul}{ }_{2} \text {, }
$$

for the optimal $k=h^{2} / \varepsilon_{m}$.

We conclude that the optimal value of $\mathrm{k}$ is given by

$$
\begin{aligned}
& k=O(h) \quad \text { if } \varepsilon_{\mathrm{m}} \leq \mathrm{Ch}, \\
& k=O\left(\mathrm{~h}^{2} / \varepsilon_{\mathrm{m}}\right) \text { if } \varepsilon_{\mathrm{m}} \geq \mathrm{Ch} .
\end{aligned}
$$

Here we notice a correspondence between the optimal value of $\mathrm{k}$ and the optimal artificial diffusion as found e.g. in Il'in's method (section 2).

Another analogy between the additional terms for $\mathrm{k} \neq 0$ and artificial diffusion for the FEM was already mentioned by Hughes and Brooks, who noticed that the 2nd order difference terms in the discrete operator $(5.8)\left(\nabla v_{h}, \bar{\varepsilon} \nabla u_{h}\right)$ and $\left(k \bar{b} \nabla v_{h}, \bar{b} \nabla u_{h}\right)$ can be combined to

$$
\left(\nabla v_{h},\left(\bar{\varepsilon}+k \bar{b}^{-T} \bar{b}\right) \nabla u_{h}\right) .
$$

Thus, to the original diffusion tensor $\bar{\varepsilon}$ an artificial term $k \bar{b}_{\bar{b}}{ }_{\bar{b}}$ is added. This diffusion acts only in the direction of $\bar{b}$, whence Hughes and Brooks call their method a Streamline-Upwind Petrov-Galerkin method. (For the relation between upwinding and artificial diffusion see section 2.)

Combining the estimates for $\varepsilon \leq h$ and $\varepsilon \geq h$, we find with the optimal

$$
\mathrm{k} \approx \frac{\mathrm{h}}{\varepsilon} \cdot \min \left(\mathrm{h}, \varepsilon_{\mathrm{m}}\right)
$$

the following error estimate

$$
\sqrt{\|v e\|_{E}^{2}+h\|\bar{b} \nabla e\|^{2}+c l e \|^{2}}<c \| u l_{2} h \sqrt{\max \left(h, \varepsilon_{m}\right)} .
$$


FURTHER DEVELOPMENTS AND BIBLIOG RAPHICAL NOTES

In the previous sections, for the 2-dimensional singular perturbation problem : only considered the Streamline-Upwind Petrov-Galerkin method by Hughes and Brooks. Irther developments and applications of this method, including time dependent prob:ms are considered by Johnson and Nävert $[36,43]$.

Two other approaches to 2-dimensional problems should be mentioned at least in a lort survey, although an extensive discussion would be somewhat off the road. The .rst is the finite difference method as devised by $\mathrm{S}$. Osher $[38,44]$. This method is ised on one-sided schemes approximating a scalar conservation law

$$
\frac{\partial u}{\partial t}=(f(u))_{x}
$$

ad can be applied to non-linear problems of the form

$$
\varepsilon y^{\prime \prime}+a(y) y^{\prime}+b(x, y)=f(x)
$$

ad its more-dimensional analogues. It is particularly suited for shock modelling ith strong shocks (interior layers and hyperbolic reduced equations).

The other method is related to multigrid methods and is studied in $[15,31,32]$. ere unstable accurate and stable inaccurate discretizations (e.g. simple central nd artificial diffusion discretization) are combined in an iterative process, to obain stable and accurate results. The advantage of this method over the above-mentiond Petrov-Galerkin and upwind methods is that there is no directional bias in the iscretization (the discretization scheme is independent of the flow-direction $\bar{b}$ ). owever, the method is not easy to implement and further research is needed before ts value is proved in practice.

\section{ibliographical notes}

In the last few years a number of books have appeared in which the numerical olution of singular perturbation problems was the main subject. We mention $[5,20,30$, $3,34,40]$. The book [20] contains a bibliography of about 200 papers. A sequel to 40], the proceedings of the BAIL II conference, will appear in 1982. Research in the ield is going on. A special issue of the journal Computer Methods in Applied Mechaics and Engineering, devoted to the topic "Optimal Finite Element Methods for Fluid lechanics and Nonsymmetric Operator Problems" is in preparation. Furthermore, in 1981 $\downarrow$ number of conferences was held at which the numerical treatment of singular perturlation problems was not the central issue, but in which several contributions were levoted to the subject. The proceedings of these conferences $[9,22,27]$ will appear in the near future. 


\section{REFERENCES}

[1] Abrahamsson, L.R., H.B. Keller and H.0. Kreiss, Difference approximations for singular perturbations of systems of ordinary differential equations, Num. Math. 22 (1974) 367-391.

[2] Ascher, U, J. Christiansen and R.D. Russe11, A collocation code for boundary-value problems, Appeared in [17].

[3] Ascher, U. and R. Weiss, Collocation for singular perturbation problems I: first order systems with constant coefficients,

Tech. Report. 81-2, Dept. Comp. Sci., Univ. BC, Vancouver, 1981.

[4] Axelsson, 0., Stability and error estimates of Galerkin finite element approximations for convection-diffusion equations,

IMA J. Num. Ana1. 1 (1981) 329-345.

[5] Axelsson, 0., L.S. Frank and A. van der Sluis, Analytical and Numerical Approaches to Asymptotic Problems in Analysis, North-Holland Pub1. Comp., Ams terdam-New York, 1981.

[6] Babuska, I. and W.C. Rheinbold, Error estimates for adoptive finite element computations, SIAM J. Numer. Anal. 15 (1978) 736-754.

[7] Babuska, I. and W.C. Rheinbold, Computational error estimates and adaptive processes for some nonlinear structural problems, Tech. Report ICMA-81-28, Univ. Pittsburg, 1981.

[8] Babuska, I. and W.G. Szymczak, An error analysis for the finite element method applied to convection diffusion problems, Tech. Note BN-962, Inst. Physical Sc. and Tech., Univ. Maryland, 1981.

[9] Baines, M.J. and K.W. Morton (eds), Proceedings of the conference on Numerical Methods in Fluid Dynamics, Reading, U.K., March 29-31, 1982.

[10] Bank, R.E. and A.H. Sherman, An adaptive Multi-Level method for Elliptic Boundary Value Problems, Computing 26 (1981) 91-105.

[11] Barrett, J.W. and K.W. Morton, Optimal finite element solutions to diffusion-convection problems in one dimension, Int. J. Num. Meth. Engng 15 (1980) 1457-1474.

[12] Barrett, J.W. and K.W. Morton, Optimal Petrov-Galerkin Methods through Approximate Symmetrization, IMA. J. Num. Anal. 1 (1981) 439-468.

[13] Barrett, K.E., Finite element analysis for flow between rotating discs using exponentially weighted basis functions, Int. J. Num. Meth. Engng 11 (1977) 1809-1817.

[14] Berger, A.E., J.M. Solomon and M. Ciment, An analysis of a uniformly accurate difference method for a singular perturbation problem, Math. Comp. 37 (1981) 79-94. 
[15] Brandt, A.,

Numerical stability and fast solutions to boundary value problems, Appeared in [40].

[16] Brooks, A.N. and T.J.R. Hughes, Streamline-Upwind Petrov-Galerkin Formulations for Convection Dominated Flows with Particular Emphasis on the Incompressible Navier-Stokes Equations, Babylon preprint series No. 5, 1981; to appear in Comp. Meth. Appl. Mech. Engng.

[17] Childs, B., M. Scott, J.W. Daniel, E. Denman and P. Nelson, Codes for Boundary Value Problems in Ordinary Differential Equations, Lecture Notes in Comp. Sc. 76, Springer Verlag, 1979.

[18] Christie, I., D.F. Griffiths, A.R. Mitchell and 0.C. Zienkiewicz, Finite element methods for second order differential equations with significant first derivatives.

Int. J. Num. Meth. Engng 10 (1976) 1389-1396.

[19] Ciarlet, P.G., The finite element method for elliptic problems, North- Holland Publ. Comp., Amsterdam-New York, 1978.

[20] Doolan, E.P., J.J.H. Miller and W.H.A. Schilders, Uniform Numerical Methods for Problems with Initial and Boundary Layers, Boole Press, Dublin, 1980,

[21] Dorr, F.W.,

The numerical solution of singular perturbations of boundary value problems, SIAM J. Num. Analysis 7 (1970) 281-311.

[22] Eckhaus, W. and E.M. de Jager, Proceedings of the conference on Singular Perturbation Theory with Applications, Oberwolfach, GFR, August 16-22, 1981. To appear in LNM series, Springer Verlag, 1982.

[23] E1-Mistikawy, T.M. and M.J. Werle, Numerical methods for boundary layers with blowing - The exponential box scheme, AIAA J. 16 (1978) 749-751.

[24] Griffiths, D.F. and J. Lorenz, An conalysis of the Petrov-Galerkin finite element method applied to a model problem, Comp. Meth. App1. Mech. Engng 14 (1978) 39-64.

[25] Groen, P.P.N. de, A finite element method with a large mesh-width for a stiff two-point boundary value problem, J. Comp. Appl. Math. 7 (1981) 3-15.

[26] Groen, P.P.N. de and P.W. Hemker, Error bounds for exponentially fitted Galerkin methods applied to stiff twopoint boundary value problems, Appeared in [33].

[27] Hackbusch, W. and U. Trottenberg, Proceedings of the Conference on Multigrid Methods, Köln, GFR, November 23-27, 1981. To appear in LNM series, Springer Verlag, 1982.

[28] Hagarty, A.F., J.J.H. Miller and E. O'Riordan, Uniform Second Order Difference Schemes for Singular Perturbation Problems, Appeared in [40].

[29] Heinrich, J.C., P.S. Huyakorn, A.R. Mitchell and O.C. Zienkiewicz, An upwind finite element scheme for two-dimensional convective transport equations,

Int. J. Num. Meth. Engng 11 (1977) 131-143.

[30] Hemker, P.W.

A numerical study of stiff two-point boundary problems, MCT 80, Mathematical Centre, Amsterdam, 1977. 
[31] Hemker, P.W., An accurate method without directional bias for the numerical solution of a 2-D elliptic singular perturbation problem,

Report NW 117/81, Mathematical Centre, Amsterdam, 1982. Appears in [22].

[32] Hemker, P.W., Mixed defect correction iteration for the accurate solution of the convection diffusion equation, Report NW 122/82, Mathematical Centre, Amsterdam, 1982. Appears in [27].

[33] Hemker, P.W. and J.J.H. Miller (eds), Numerical Analysis of Singular Perturbation Problems, Academic Press, London, 1979.

[34] Hughes, T.J.R. (ed.), Finite Element Methods for Convection Dominated Flows, ASME, New York, 1979.

[35] $\mathrm{Il}$ 'in, A.M., Difference scheme for a differential equation with a small parameter affecting the highest derivative, Mat. Zametki 6, 237-248; Math. Notes 6 (1969) 596-602.

[36] Johnson, C. and U. Nävert, Analysis of some finite element methods for advection-diffusion problems, Appeared in [5].

[37] Kellogg, R.B. and A. Tsan, Analysis of some difference approximations for a singular perturbation problem without turning points,

Math. Comp. 32 (1978) 1025-1039.

[38] Lorenz, J., Nonlinear singular perturbation problems and the Engquist-Osher difference scheme,

Report 8115, Math. Inst., Univ. Nijmegen, 1981.

[39] Mi11er, J.J.H.,

Sufficient conditions for the convergence, uniformly in $\varepsilon$, of a three-point difference scheme for a singular perturbation problem,

In: Numerical Treatment of Differential Equations in Applications ( $R$. Ansorge and W. Tornig, ed.), Lect. Notes in Maths. No. 679 (1978) 85-91, Berlin, Springer.

[40] Miller, J.J.H. (ed.), Boundary and Interior Layers-Computational and Asymptotic Methods, Boole Press, Dublin, 1980.

[41] Miranker, W.L. and D.Y. Yun, A note on boundary Zayer elements, Report RC 4990, IBM Yorktown Heights, 1974.

[42] Morton, K.W.,

Finite element methods for non-selfadjoint problems,

Num. Anal. Rept 3/81, Univ. Reading; To appear in: Procs SRC Numerical Analysis Sumer School and Workshop, Univ. Lancaster, to be published by Springer Verlag.

[43] Nävert, U., The stream-line diffusion method for time dependent convection-diffusion problems with small diffusion, Report 81.01.R, Chalmers Univ. Tech., Göteborg, 1981.

[44] Osher, S.,

Nonlinear singular perturbation problems and one-sided difference schemes, SIAM J. Numer. Ana1. 18 (1981) 129-144.

[45] Pearson, C.E.,

on a differential equation of boundary layer type,

J. Math. Phys. 47 (1968) 134-154. 
[46] Pereyra, V.,

PASVA 3: an adaptive finite difference program for first order nonlinear ordinamy boundary problems, Appeared in [17].

[47] Raithby, G.D., A critical evaluation of upstream differencing applied to problems involving fluid flow, Comp. Meth. App1. Mech. Engng 9 (1976) 75-103.

[48] Reinhardt, H.J., A-posteriori error estimates and adoptive finite element computations for singularly perturbed one space dimensional parabolic equations, Appeared in [5].

[49] Reinhardt, H.J.,

$A$-posteriori error estimates for the finite element solution of a singularly perturbed linear ordinary differential equation,

SIAM J. Num. Analysis 18 (1981) 406-430. 